\title{
Behavioral Consistency Extraction for Face Verification
}

\author{
Hui Fang and Nicholas Costen \\ Manchester Metropolitan University \\ Department of Computing and Mathematics, \\ Manchester, U.K. \\ $\{$ H.Fang, N. Costen $\} @ m m u$. ac.uk
}

\begin{abstract}
In this paper we investigate how the use of computational statistical models, derived from moving images, can take part in the face recognition process. As a counterpart to psychological experimental results showing a significant beneficial effect of facial non-rigid movement, two features obtained from face sequences, the central tendency and type of movement variation, are associated to improve face verification compared with single static images. By using General Group-wise Registration algorithm, the correspondences across the sequences are captured to build a combined shape and appearance model, parameterizing the face sequences. The parameters are projected to an identity-only space to find the central tendency of each subject. In addition, facial movement consistencies across different behaviors exhibited by the same subjects are recorded. These two features are fused by a confidence-based decision system for authentication applications. Using the BANCA video database, the results show that the extra information extracted from moving images significantly and efficiently improves performance.
\end{abstract}

\section{Introduction}

In recent decades, a great deal of attention has been paid to modeling static face recognition. A large number of algorithms, including Principle Components Analysis (PCA) [1, Elastic Bunch Graphic Matching 2], Independent Component Analysis [3], kernel-based algorithms [4, Linear Discriminant Analysis (LDA) 5] and Active Appearance Model (AAM) [ 6 have been developed in an attempt to model the recognition process. Although face identification systems based on them have achieved high level of performance in standardized face recognition or verification tests such as FERET [7, FRVT [8] and BANCA [9], and the combined processing of a number of images of the same person is known to very significantly reduce recognition errors [10, it is still expected that performance will be improved by the use of video sequences rather than from still images.

At the same time, psychological evidence has been accumulating, which indicates that the dynamic information available from moving faces also makes a significant contribution to face recognition. There are three major, non-exclusive hypotheses about the role of facial movements in face recognition [11. The first

A. Esposito and R. Vích (Eds.): Cross-Modal Analysis, LNAI 5641, pp. 291305 2009.

(C) Springer-Verlag Berlin Heidelberg 2009 
depends on the observation that the 3D structure of the face can be recovered via the Structure-from-Motion (SfM) algorithm. The second suggests that robust confirmation by multiple frames is possible in poor viewing conditions (together these are referred to as "structural enhancement") and the third uses "characteristic motion patterns" to distinguish individual faces. Based on these psychological findings, it is interesting to see if computer vision can exploit similar additional information from moving images to help in modeling computational recognition and improve recognition performance.

The application of the Structure-from-Motion algorithm 12 to face modeling is an ill-posed problem, although a number of non-rigid SfM algorithms 13, 14 have been proposed. SfM assumes the object undergoes relatively large rigid movements compared with small deformations. However, this is implausible with faces, as large rotations induce self-occlusions. This means the correspondences between parts cannot be tracked consistently, breaking the fundamental assumptions of the algorithm. In addition, the non-rigid movement of the face (as when the subject speaks or shows emotions) further degrades the 3D structure recovered. One feasible solution [15] is to build 3D face models by acquiring data from 3D scanner devices and parameterizing the face instance by reducing the projection error to the sequence. However, this would be highly dependent on the 3D data acquisition process.

In this paper, we investigate the other two factors mentioned in the psychological experiments. A generic combined 2D Appearance Model (AM) [6] is built from the ensemble (the set of faces used to provide the system with generic facial knowledge) using correspondences obtained from the General Group-wise Registration algorithm (GGR). The use of the AM is known to improve facial representation and recognition relative to systems which do not explicitly model face shape [16. All the facial instances are parameterized on this model and the consistency of the parameters is explored, projecting onto an identity sub-space (obtained by LDA performed on the ensemble), using sequences of the same and different subjects. This yields representative measures of the central tendencies for identity parameters. At the same time, the common characteristic motions of the same subjects are extracted by finding a maximally correlated sub-set of the eigenvectors derived from the parameters of a single sequence. Finally, the features extracted from moving faces are fused by a reliability-based framework to verify the true clients. Through the experiments, a conclusion in accordance with psychological work by Lander et al. [17] is obtained, which shows that the benefits of moving faces are mainly from non-rigid movements.

The originalities of our contributions can be highlighted as: (1) General Groupwise Registration (GGR) algorithm to extend combined appearance model from modeling static image to moving images; (2) a new way to represent the type of moving variations of the same subjects; (3) better recognition from moving images which is accordant with the corresponding psychological experimental results by fusing the two types of moving information.

This paper is structured as follows. In the next section the relevant work is introduced and some differences between them and the proposed algorithm are 
mentioned. In section 3, the method used is presented in detail. Experimental results are shown in section 4 . Finally in section 5 conclusions are drawn and future work is introduced.

\section{Relevant Work}

Normally a tracking and recognition scheme is used for face recognition in video sequence. Zhou et al. [18 investigate a probabilistic approach to video-based recognition by using a particle filter. In principle, this algorithm assumes that the identity of a person in a sequence is constant and searches for the maximum probability solution. Although this is similar to the central tendency concept in our proposed algorithm, the motion patterns were not explored in this paper.

A similar system was proposed by Benedikt et al [19, using high-speed 3D videos of short sequences of speaking. After parameterization by Principal Component Analysis, maximum discrimination between individuals was obtained using Dynamic Time Warping; this was significantly better than a Hidden Markov Model (HMM), perhaps because of the relatively small amount of data available. It should be noted that in this case, all of the sequences were restricted to the same behaviour (saying the word "Puppy").

Liu and Chen 20. present a HMM based algorithm which finds the highest score of the state transition probability from the facial motion in the sequence. They project the moving images into a PCA subspace and define states from which to train the HMM. Although the transitions between these states represent the motion patterns of the subjects to some extent, it is quite difficult to define the discrete states and quantize the parameters of the images.

Combined shape and texture model has been used for analyzing the face motion by AAM. Bettinger et al. 21] present a generative model of facial behavior. All of the faces sequences are registered by AAM and are projected into a combined shape and texture model. Fragments of sequences with the same behavior, such as shaking the head or smiling, are grouped, followed by modeling using a variable length Markov model to find the temporal relationships. This work assumes that same facial behaviors of different subjects share the same characteristics while we are seeking consistencies across different behaviors, such as speaking different sentences, exhibited by the same subjects. In addition, as with Liu and Chen 20, very considerable sequence lengths are required to allow effective training of the Markov models; these are not available here. We also find that the GGR algorithm is more suitable for solving the correspondence problem for registration tasks where a large diverse data set is used.

Edwards et al. 22 propose an online scheme of learning a linear relationship between the parameters in an identity space and its non-identity space, embedded within a shape and texture model space. These relationships are used to correct the identity parameters, improving the recognition from video sequences. The framework of our proposed algorithm is similar; however we find the motion patterns of subjects directly in the non-identity space and fuse the features in identity space with it for recognition instead of looking for the relationship 
between them. Our scheme is more suitable to find similarities of the patterns when presented with different behaviors in relatively short sequences and gives a much clearer perspective to connect with psychological experiments.

Yamaguchi et al. 23] report a system similar to that proposed here, although using an eigenface encoding. Relatively long sequences, where the predominate form of variation involves facial orientation, are encoded and the similarity of their distributions are compared using a Mutual Subspace Method (MSM), measuring the relative angle at which they lie within a larger space. This was extended by Arandjelović and Cipolla 24] and notably high recognition performance was observed when mean face encoding was also included, but this depended upon the use of a procedure which compensates for different proportions of facial orientations in the sequences by physical modeling these changes. It is unclear whether this latter process could be applied to sequences where the major variations are plastic facial changes (typically expressive or speech deformations).

\section{Proposed Algorithm}

In the proposed framework, the two factors mentioned in the psychological experiments, structural enhancement and characteristic motion patterns are simulated in computer vision. The two correspondences, named as identity central tendency and principle component based motion patterns are fused by a confidence-based system to synthesize the improvement in human face perception when presented with video sequences rather than static images. The overall paradigm is shown in Figure 1. It should however be noted that there are potentially other effects dependent upon the order in which sequence frames are presented 25, which will not be simulated in this study.

\subsection{General Group-Wise Registration}

Pair-wise (template-based) registration is a traditional technique for finding the correspondences in a group of images. However, it is unlikely that the reference image represents the whole set of images best; this makes the corresponded structures unreliable. A group-wise registration scheme may improve performance by expanding the set of references.

GGR is an automatic registration algorithm which finds the correspondences across a set of images. It shares similar ideas with others [26, 27] which seek to model sets efficiently, representing the image set and iteratively fitting this model to each image. Operational details can be found elsewhere 28.

The implementation of the group-wise registration has a number of steps. First, one image is selected as a reference template and all other images are registered using a traditional template match. Next, a statistical shape model and texture model is build to represent the image set. Each image is represented in the model and the correspondences are refined by minimizing a cost function. Finally the statistical models are undated and the fitting repeated until convergence.

The model used in this paper is a simple mean shape and texture built by warping all the faces to the mean shape using a triangular Delauney mesh. 


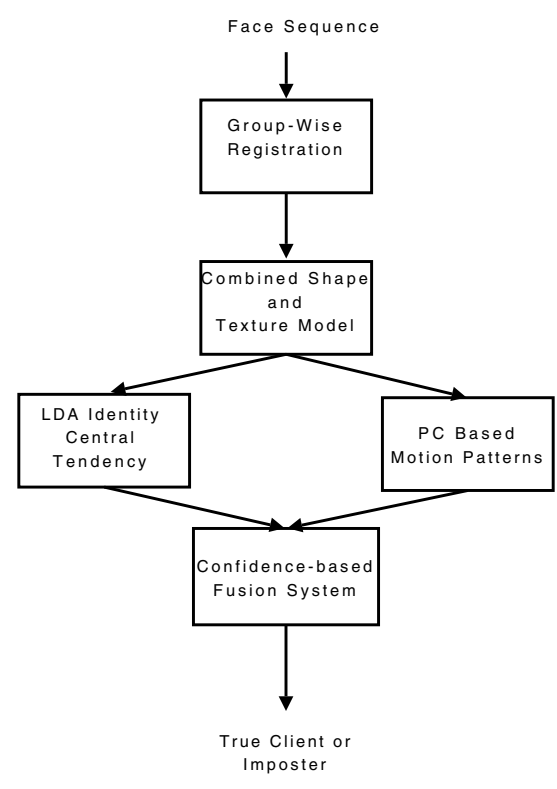

Fig. 1. The stages of processing on a sequence to reach an identity decision

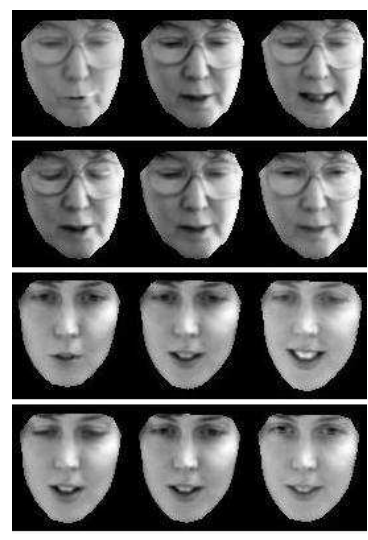

Fig. 2. Varying $+/-3 s . d$. from individual subject means two significant modes

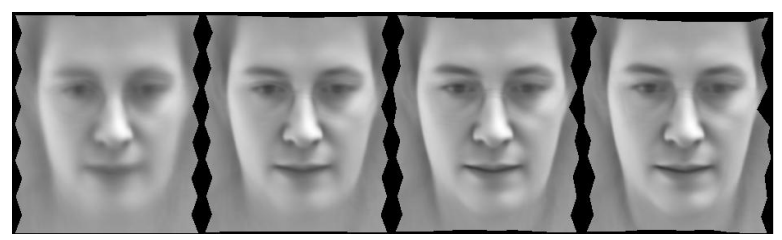

Fig. 3. Representative stages of the iterated updating of the mean face model for a single individual. Later stages are to the right; note the increased level of detail.

Figure 3 shows how the registration improves and the model represents the face structure more accurately as different subjects are progressively aligned together. The matching step deforms each image by minimizing a cost function. A coarse-to-fine scheme is applied to increase the number of control points and optimize their position. In the final iterations, the points are moved individually to minimize the cost. The cost function includes both shape and texture parts,

$$
E=\lambda \sum_{i}\left(c-\frac{0.5\left\|d_{i}-\left(\Delta d_{i}+d_{n e i g}\right)\right\|}{\sigma_{s}^{2}}\right)-\frac{|r|}{\sigma_{r}}
$$

where $r$ is the residue between the model and the current image after deformation, $\sigma_{r}$ and $\sigma_{s}$ are the standard deviations of the residue and shape, $c$ is a constant, $d_{i}$ is the position of the $i^{t h}$ control point, $d_{n e i g}$ is the average position 
of the neighborhood around point $i$ and $\Delta d_{i}$ represents the offset of the point from the average mean shape.

The initialization of the sparse correspondent feature points is set manually on the mean image of the image set. When GGR finds the dense correspondences across the images, all the sparse feature points are warped to each image using the triangulation mesh. This helps avoid errors made by the correspondence matching step and allows concentration upon investigating the importance of the motion patterns.

It should be noted that while GGR is a possible description of the process of consolidating faces, with its repeated iteration across the sequence, it is not necessarily an adequate description of the perception of new face in a sequence. One possible model of this process centres on building a model of the variation within the sequence, which then constrains the interpretation of the next frame 29. It is notable that this may also provide an explanation of the effects of jumbling frame-order in the image-sequence.

\subsection{Combined Shape and Texture Model}

The faces are encoded using an Appearance Model [6]; this takes the output of the GGR and approximates the manifold, or high dimensional surface, on which the faces lie. This allows accurate coding, recognition and reproduction of previously unseen examples. Pixels defined by the GGR points as part of the face are warped to a standard shape, ensuring that the image-wise and face-wise coordinates of images are equivalent. If a rigid transformation to remove scale, location and orientation effects is performed on the point-locations, they can then be treated in the same way as the grey-levels, as again identical values for corresponding points on different faces will have the same meaning.

Redundancies between feature-point location and grey-level values can be removed and dimensionality estimated by Principal Components Analysis. Given a set of $N$ vectors $\mathbf{q}_{i}$ (either the pixel grey-levels, or the feature-point locations) sampled from the images, the covariance matrix $\mathbf{C}$ of the images is calculated,

$$
\mathbf{C}=\frac{1}{N} \sum_{i=1}^{N}\left(\mathbf{q}_{i}-\overline{\mathbf{q}}\right)\left(\mathbf{q}_{i}-\overline{\mathbf{q}}\right)^{T},
$$

and orthogonal unit eigenvectors $\boldsymbol{\Phi}$ and a vector of eigenvalues $\lambda$ are extracted from $\mathbf{C}$.

Redundancies between configuration and pigmentation are removed by performing separate PCAs upon the shape and grey-levels, providing shape parameters $\mathbf{w}_{\mathbf{s} i}$ and texture parameters $\mathbf{w}_{\mathbf{t} i}$. These are combined to form a single vector for each image on which second PCA is performed [30]. This gives a single feature vectors

$$
\mathbf{x}=\boldsymbol{\Phi}_{\mathbf{c}}{ }^{T}\left[\begin{array}{c}
\mathbf{W}_{\mathbf{s}} \boldsymbol{\Phi}_{\mathbf{s}}{ }^{T}\left(\mathbf{q}_{\mathbf{s}}-\overline{\mathbf{q}}_{\mathbf{s}}\right) \\
\boldsymbol{\Phi}_{\mathbf{t}}^{T}\left(\mathbf{q}_{\mathbf{t}}-\overline{\mathbf{q}}_{\mathbf{t}}\right)
\end{array}\right]
$$

for each image, assuming zero-mean weights and where $\mathbf{W}_{\mathbf{s}}$ is a diagonal vector of weights compensating for the characteristic scales of the shape and texture 
parameters. This 'appearance model' allows the description of the face in terms of true, expected variation [16].

\subsection{Central Tendency}

In the same sequence, regardless of parameter change due to different poses, lighting and expressions, the representation of the identity can be expected to be constant. Stabilization of representation by taking the mean of a number of samples is known to improve recognition performance [10. However, in this case, the model will encode (even after averaging) both identity and non-identity variation. To remove the latter, a Linear Discriminate Analysis subspace [5] is used ensure that only identity information is encoded. LDA is a linear subspace calculated from the $n$ frames available of each of the $p$ individuals in the ensemble, using

$$
\mathbf{C}_{B}=\frac{1}{p} \sum_{i=1}^{p}\left(\overline{\mathbf{x}}_{\mathbf{i}}-\overline{\mathbf{x}}\right)\left(\overline{\mathbf{x}}_{\mathbf{i}}-\overline{\mathbf{x}}\right)^{T}
$$

and

$$
\mathbf{C}_{W}=\frac{1}{n p} \sum_{i=1}^{p} \sum_{j=1}^{n}\left(\mathbf{x}_{\mathbf{i j}}-\overline{\mathbf{x}}_{\mathbf{i}}\right)\left(\mathbf{x}_{\mathbf{i j}}-\overline{\mathbf{x}}_{\mathbf{i}}\right)^{T}
$$

to give

$$
\mathbf{C}_{D}=\mathbf{C}_{B} \mathbf{C}_{W}^{-1} .
$$

Eigenvectors are then taken to provide a subspace which maximizes variation between individuals and minimizes that within them. Each frame in a sequence is projected onto this subspace to give a descriminative encoding $\mathbf{d}$, before taking the mean and assessing similarity with other sequences,

$$
S_{c}=\frac{\overline{\mathbf{d}}_{i}}{\left|\overline{\mathbf{d}}_{i}\right|} \cdot \frac{\overline{\mathbf{d}}_{j}}{\left|\overline{\mathbf{d}}_{j}\right|} .
$$

\subsection{Motion Patterns}

When the moving images in one sequence are projected into the combined shape and appearance subspace, the variation of these encoded representations reveal the movement of the face; given the psychology it should be possible to perform recognition on this variation. The characteristic motion patterns are captured by using PCA performed upon all of the encodings $\mathbf{x}$ of a single sequence to yield a single set of eigenvectors $\boldsymbol{\Phi}$. Although there is no direct relationship between the motion patterns (a series of eigenvectors) and the specific behaviors of faces, some of the more important of variations actually represent some meaningful changes. Examples for two sequences are shown in Figure 2, In this figure, the images are synthesized by adding 3 standard deviations of change on two modes of the individuals; they are obviously related to some kinds of mouth and eye orientation changes. 
The similarity of pairs of spaces can be compared as

$$
S_{m}=\max \left(\operatorname{trace}\left(\operatorname{colperm}\left(\boldsymbol{\Phi}_{\mathbf{i}} \cdot \mathbf{\Phi}_{\mathbf{j}}^{T}\right)\right)\right),
$$

where it should be noted that the ordering of the components extracted by the PCA will be determined by their associated eigenvalues and so the value of $S_{m}$ is liable to be reduced by variation in the magnitude of different behavioural tendencies in different sequences of the same individual. This is overcome here by permuting the columns of the matrix describing the relationship between any two sequences before taking the trace so as to maximize $S_{m}$. It should be noted that this will involve calculating different permutions for a single probe sequence when comparing it with multiple gallery members. In addition, $\boldsymbol{\Phi}_{\mathbf{i}}$ and $\boldsymbol{\Phi}_{\mathbf{j}}$ may be (identically) truncated to remove noisy, low-variance eigenvectors.

\subsection{Fusion of Facial Identity Characteristics}

Although recognition may be performed on both the central tendency and motion patterns independently, neither shows perfect performance and it is possible to combine the two, balancing one against another. A confidence-based fusion system [31] is adopted, based on how definite is each type of information. The fused similarity can be calculated,

$$
S_{f}=\frac{C_{S c}}{C_{S c}+\alpha C_{S m}}\left(\frac{S_{c}-\mu_{S c}}{\sigma_{S c}}\right)+\frac{\alpha C_{S m}}{C_{S c}+\alpha C_{S m}}\left(\frac{S_{m}-\mu_{S m}}{\sigma_{S m}}\right) .
$$

Here $\mu$ and $\sigma$ are the average and standard deviation of the similarity measures, allowing normalization. $C$ is a confidence value associated with the two type of information, shown in Figure 4 and is calculated as the absolute difference between the False Acceptance and False Rejection rates; this is dependent upon the similarity between probe and gallery for that information type and gives a greater weighting to parameters which will yield confident decisions to either accept or reject and individual. Finally $\alpha$ is a weight value, trading between

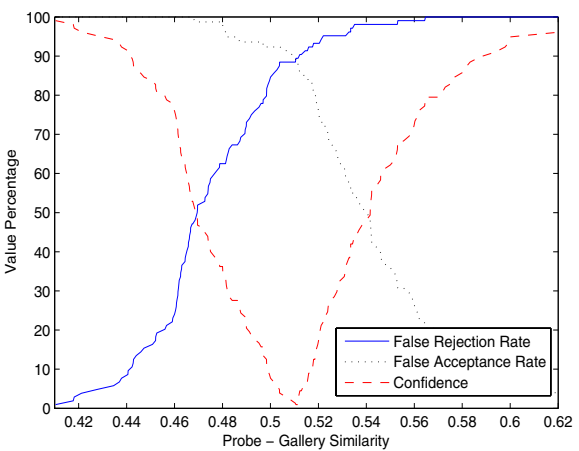

Fig. 4. The confidence of motion pattern based on the FA and FR rates

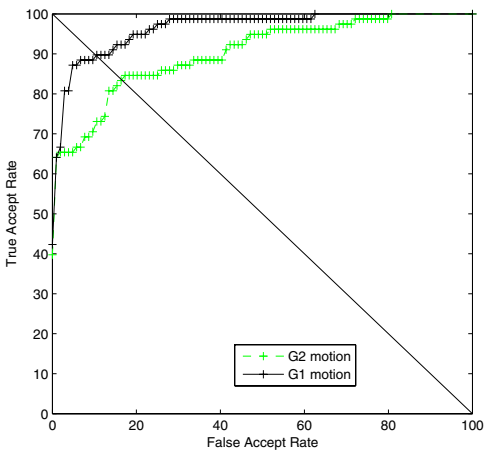

Fig. 5. ROC curves for motion patterns with 41 eigenvectors 
the central tendency and movement measures. All of these parameters can be derived from a set of calibration sequences.

\section{Experiment}

\subsection{Setup}

We evaluate the framework by testing performance for facial verification. This is an application of face recognition which seeks to determine whether an individual's real and claimed identity (as determined by some other means such as a password, identity card, or, as here, a spoken name) coincide. Thus, centering on one-to-one matches, it differs from facial familiarity or identification. Both of these involve one-to-many matches but the latter requires recording which gallery face is most similar to the probe, while the former concerns the degree of similarity to any and all of the gallery. The same features and combination methods could be used in these cases.

The BANCA database used here includes 52 subjects, half male and half female, each of which (verbally) claims the identity of two individuals four times (thus each individual can be used as both a true client and an imposter). Each such claim sequence has approximately 500 frames of frontal images, of which 4 representative frames of one individual are shown in Figure 6. The database is divided into two groups, $g 1$ and $g 2$, intended for a leave-some-out design. The 26 subjects in $g 1$ and an extra 10 subjects (so totaling 36 subjects) form an ensemble and are used to build model, which allows testing of the encoding of the 26 subjects in $g 2$ and vice versa. This both avoids overfitting of the model (individuals to be recognized are not present in the ensemble) and un-representativeness of the model (faces swap between ensemble and gallery). One of the correct claims for each test individual is used in the gallery (those frame which are encoded to give specific knowledge about particular, identified individuals), all the other claims are probes and are so used to test recognition performance.

Because of the size of the database (about 20 seconds for each person, thus over 250,000 images in the ensemble), representative frames are selected for each ensemble subject by building an appearance model of that individual, encoding

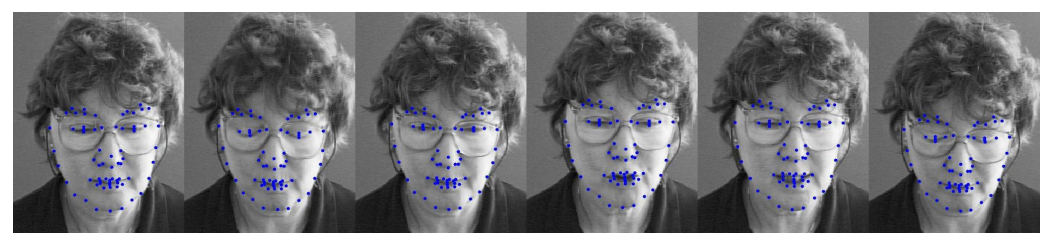

Fig. 6. Representative frames from a BANCA individual. The left most image is the first frame, used the "static image" condition. The images have the landmark positions indicated on them; these are not present when the texture is processed by the algorithm. 
each fame on this AM and using k-means clustering to divide the encodings into approximately 10 groups (one for each 50 frames). The frames most representative for each group are then selected and used to build both the combined AM and LD model.

The model yields a similarity value for each probe and its claimed identity, which will be either a True Entry or an Imposter Attack. Across the entire set of probes, each similarity can be used as a threshold, so generating a Receiver Operating Characteristic (ROC) curve, which summarizes its performance by trading between the False Acceptance (FA) and False Rejection (FR) rates as shown in Figures 5 , 9and10, Two performance criteria, Equal Error Rate (EER) and the Area Under the Curve (AUC) are used to present the results. In a typical authentication application, the EER is the best tradeoff, at the point of equal FA and FR. However, this parameter only concerns a single point on the ROC curve, and so is not necessarily representative of all changes (particularly those associated with the extremes of FA and FR). AUC overcomes this, measuring the total discrimination, and ranging from 1 (for perfect discrimination) to 0 (for perfect reversed discrimination). If building an actual application, an individual threshold can be chosen based on the relative risks of the two types of error.

\subsection{Experimental Results}

All of the probe and gallery frames are encoded on the world combined AM and LD model and are used to derive the central tendency and motion pattern parameters. We evaluate the performance of the static verification system by two means of selecting images. Firstly, we selecting the very first frame from each gallery and probe sequence and encode those alone, on the $S_{C}$ measure. Secondly, we randomly select one image from each sequence and encode those.

Motion Parameters. The individual eigenmodels which provide the motionpattern parameters are each derived from approximately 500 observations, each of which has 41 dimensions. The lower of these figures limits the number of parameters which can be derived from the PCA, but some, particularly those with small eigenvalues may be spurious. The effects of varying the number of parameters extracted from the gallery images (different lines) and the number of probe-gallery parameter pairings to include in the similarity measure (the axilla) for AUC on g2 are shown in Figure 7, There is no noticeable interaction between the means of controlling the number of parameters, but performance continues increasing until the maximum is reached. This pattern is probably an unfortunate consequence of the relatively small ensemble (36 individuals) and the high similarity between gallery and probe sequences. It is possible that with more variation between the gallery and probes some significant truncation would be required. For comparison purposes, the AUC for the MSM similarity 23 is also plotted; this is significantly lower. Quantitative comparisons for the two groups, with 41 eigenvectors are shown in Table 1, including two-tailed probability differences 32 . 
Table 1. Error rates and AUCs for movement using the proposed algorithm and MSM. Probabilities are two-tailed paired-sample tests of similarity of the AUCs.

\begin{tabular}{|l|cc|ccc|ccc|}
\hline & \multicolumn{2}{|c|}{ EER } & \multicolumn{2}{|c|}{ AUC g1 } & \multicolumn{2}{c|}{ AUC g2 } \\
\hline Data Type & g1 & g2 & AUC & Z & Prob & AUC & Z & Prob \\
\hline Motion Patterns & 10.50 & 16.43 & 0.9072 & \multirow{2}{*}{3.71} & $\mathbf{0 . 0 0 0 2}$ & 0.9644 & \multirow{2}{*}{4.82} & $\mathbf{0 . 0 0 0 1}$ \\
MSM & 29.84 & 28.24 & 0.7563 & & & 0.8061 & & \\
\hline
\end{tabular}

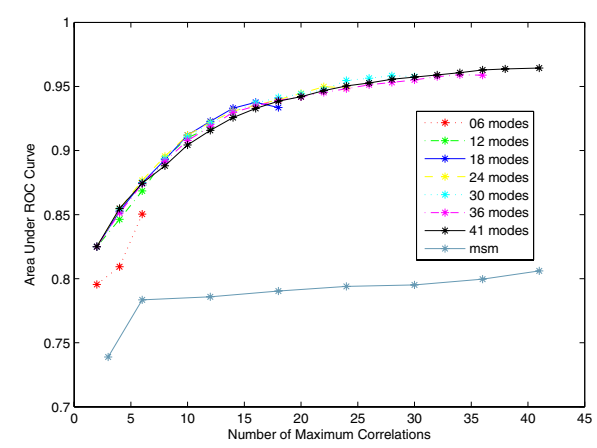

Fig. 7. Variation in motion-pattern AUC with gallery and probe dimension

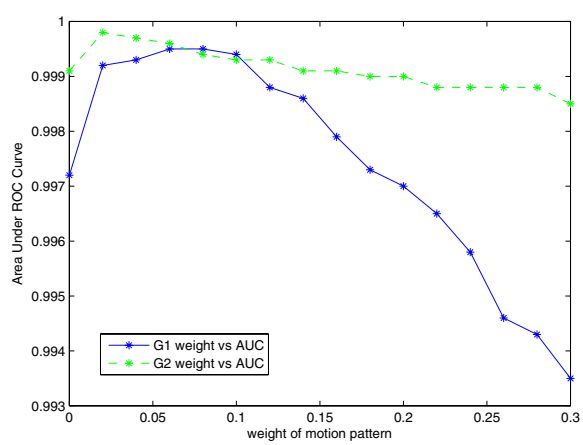

Fig. 8. Variation in combined AUC motion-pattern weighting function

Parameter Fusion Weighting. Although the confidence measures and means and standard deviations can be simply read from the similarity values of the calibration set ( $g 1$ for $g 2$ verification and vice versa), the weight value $\alpha$ must be selected given the classification performance of the fused parameters. The AUC measure for the two groups of sequences is shown in Figure 8. Note that a weight of 0 implies the use of central tendency alone, and that an $\alpha$ value in the range $0-$ 0.2 improves classification performance (the final weighting is set to 0.08 for both groups). This relatively low importance of movement is understandable, given the relatively low discrimination shown by the motion patterns (all 41 eigenvectors) in Figure 5, and corresponds with the relative difficulty humans find in learning or recognizing faces from motion alone, without grey-level information [33.

Combined Recognition. The overall results for the two groups are shown in Figures 9 and 10 (note that these only show the top 15\%), while the EER and AUC values are given in Table 2, including one-tailed probabilities of significant differences from the "Random Image" condition. In both cases, the combined central tendency and motion pattern gives a much lower EER and higher AUC than the static images, with intermediate performance on the central tendency. As can be seen from Figure 5, performance on the motion alone is much weaker. The variation in the results between the two groups suggests that while the initial frames for $g 1$ are unusually representative, there are more significant consistencies in the motion than are seen in $g 2$, which probably lacks motion variation 


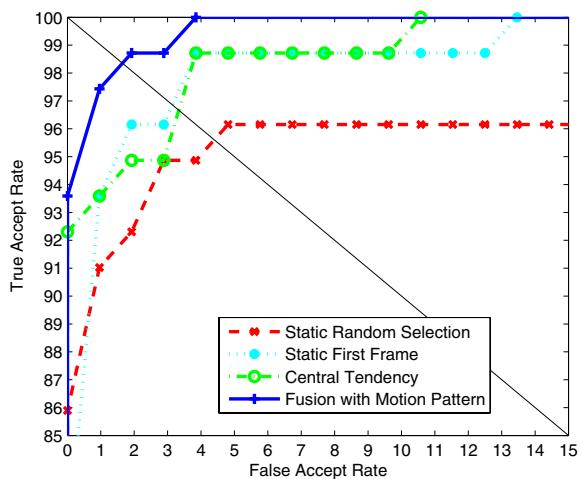

Fig. 9. ROCs for group 1 images

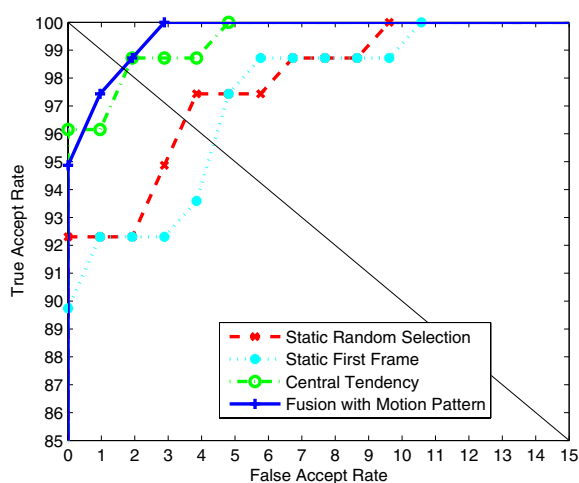

Fig. 10. ROCs for group 2 images

Table 2. Error rates and AUCs for two sorts of static images, central appearance tendency and fusion of the sequential information. Probabilities are one-tailed pairedsample tests of similarity of the AUCs relative to the "Random Frame" condition, as we are consistently adding information to the system.

\begin{tabular}{|l|cc|ccc|ccc|}
\hline & EER & \multicolumn{3}{|c|}{ AUC g1 } & \multicolumn{3}{c|}{ AUC g2 } \\
\hline Data Type & g1 & g2 & AUC & Z & Prob & AUC & Z & Prob \\
\hline Random Frame & 4.41 & 3.94 & 0.9869 & & & 0.9965 & & \\
First Frame & 3.29 & 4.65 & 0.9965 & 1.6657 & $\mathbf{0 . 0 4 8 5}$ & 0.9958 & 0.4328 & 0.3336 \\
Central Tendency & 3.93 & 1.92 & 0.9972 & 1.7774 & $\mathbf{0 . 0 3 7 5}$ & 0.9991 & 1.6988 & $\mathbf{0 . 0 4 5 5}$ \\
Fusion of motion & 1.68 & 1.68 & 0.9995 & 1.9063 & $\mathbf{0 . 0 2 8 1}$ & 0.9994 & 1.6250 & 0.0516 \\
\hline
\end{tabular}

within the sequences. Thus the static images are slightly easier to recognize and so the difference with combined recognition is (just) non-significant. This appears to be a peculiarity of the (random but gender-balanced) selection of sequences in the two groups.

\section{Conclusions and Future Work}

We have described a framework to use the motion information in facial sequences to improve face authentication. Two kinds of motion-derived features, the mean parameters in identity space and major correlated variations across the sequences of the same subjects, are captured and fused to allow verification of the identity of the face. The associated features from the moving faces achieve significantly better performance than using the static images. In addition, the motion patterns themselves are significantly more useful than the comparable MSM measure [23]. One interpretation of this matrix permution effect is that we are reproducing the ballencing effect of repopulating the face-space 24], without the need to use a physical model to hypothesize views. Thus we can extend this advantage into situation where most of the motion is plastic deformation, as here. The 
fusion technique allows an automatic trade-off between the use of appearance and motion-based information as a function of the composition of the dataset. All of the parameters (with the partial exception of the relatively stable mean motion weighting factor) are determined directly from the dataset. However it should be noted that the need to use all of the possible dimensions in the motion variation features to get peak performance does suggest a degree of overfitting imposed by the design of the dataset. Different sets of sequences and different recognition protocols will yield slightly different patterns of results but should not alter their general tenor.

The results also reflect how faces are recognized by the human cognitive system when presented with video sequences. As the cognitive system accumulates the stabilized appearance information and characteristic motion from the sequence, it predominately recognizes the face based on the appearance. However, if the appearance features are indistinct but the confidence in the characteristic motion patterns is high, the decision will be made based on the latter feature. This is a possible reason why face recognition can be improved using video sequences in both human and computer vision.

In future work, we will investigate how the characteristic motion patterns can be expanded to include explicit information on the sequence of facial configurations and test the framework by direct comparison with human performance.

\section{Acknowledgments}

We would like to thank Prof. Tim Cootes and Prof. Chris Taylor of Manchester University for providing comments and helpful suggestions. This work was supported by EPSRC grants EP/D056942 and EP/D054818.

\section{References}

[1] Turk, M., Pentland, A.: Face recognition using eigenfaces. In: Computer Vision and Pattern Recognition Conference, pp. 586-591 (1991)

[2] Wiskott, L., Fellous, J.M., Knueuger, N., Malsburg, C.: Face recognition by elastic bunch graph matching. IEEE Transations on Pattern Analysis and Machine Intelligence 19(7), 775-779 (1997)

[3] Bartlett, M., Movellan, J., Sejnowski, T.: Face recognition by independent component analysis. IEEE Transations on Neural Networks 13(6), 1450-1464 (2002)

[4] Lu, J., Plataniotis, K., Venetsanopoulos, A.: Face recognition using kernel direct discriminant analysis algorithms. IEEE Transations on Neural Networks 14(1), 117-126 (2003)

[5] Belhumeur, P.N., Hespanha, J.P., Kriegman, D.J.: Eigenfaces vs. Fisherfaces: Recognition using class specific linear projection. IEEE Transations on Pattern Analysis and Machine Intelligence 19, 711-720 (1997)

[6] Cootes, T.F., Edwards, G.J., Taylor, C.J.: Active appearance models. IEEE Transations on Pattern Analysis and Machine Intelligence 23(6), 681-685 (2001)

[7] Phillips, P., Moon, H., Rizvi, S., Rauss, P.: The feret evaluation methodology for face recognition algorithms. IEEE Transations on Pattern Analysis and Machine Intelligence 22(10), 1090-1104 (2000) 
[8] Phillips, P., Flynn, P., Scruggs, T., Bowyer, K., Chang, J., Hoffman, K., Marques, J., Min, J., Worek, W.: Overview of the face recognition grand challenge. In: Computer Vision and Pattern Recognition Conference, pp. 947-954 (2005)

[9] Bailly-Bailliere, E., Bengio, S., Bimbot, F., et al.: The banca database and evaluation protocol. In: International Conference on Audio- and Video-Based Biometric Person Authentication, pp. 625-638 (2003)

[10] Jenkins, R., Burton, A.M.: $100 \%$ accuracy in automatic face recognition. Science 319(5862), 435 (2008)

[11] O'Toole, A., Roark, D., Abdi, H.: Recognizing moving faces: A psychological and neural synthesis. Trends in Cognitive Sciences 6(6), 261-266 (2002)

[12] Tomasi, C., Kanade, T.: Shape and motion from image streams under orthography: a factorization method. International Journal of Computer Vision 9(2), $137-154$ (1992)

[13] Torresani, L., Hertzmann, A., Bregler, C.: Learning non-rigid 3D shape from 2D motion. In: Neural Information Processing Systems, pp. 1555-1562 (2003)

[14] Xiao, J., Chai, J.X., Kanade, T.: A closed-form solution to non-rigid shape and motion recovery. International Journal of Computer Vision 67(2), 233-246 (2006)

[15] Blanz, V., Vetter, T.: Face recognition based on fitting a 3D morphable model. IEEE Transations on Pattern Analysis and Machine Intelligence 25(9), 1-12 (2003)

[16] Craw, I.G., Costen, N.P., Kato, T.: How should we represent face for automatic recognition? IEEE Transactions on Pattern Analysis and machine Inteligence 21(8), 725-736 (1999)

[17] Lander, K., Chuang, L.: Why are moving faces easier to recognize? Visual Cognition 23(3), 429-442 (2005)

[18] Zhou, S., Krueger, V., Chellappa, R.: Probabilistic recognition of human faces from video. Computer Vision and Image Understanding 91, 214-245 (2003)

[19] Benedikt, L., Kajic, V., Cosker, D., Rosin, P.L., Marshall, D.: Facial dynamics in biomedtric identification. In: British Machine Vision Conference, pp. 1065-1075 (2008)

[20] Liu, X., Chen, T.: Video-based face recognition using adaptive hidden markov models. In: Computer Vision and Pattern Recognition Conference, pp. 26-33 (2003)

[21] Bettinger, F., Cootes, T., Taylor, C.: Modelling facial behaviours. In: British Machine Vision Conference, pp. 797-806 (2002)

[22] Edwards, G., Taylor, C., Cootes, T.: Improving identification performance by integrating evidence from sequences. In: Computer Vision and Pattern Recognition Conference, pp. 1486-1491 (1999)

[23] Yamaguchi, O., Fukui, K., Maeda, K.: Face recognition using temporal sequence. In: IEEE International Conference on Automatic Face and Gesture Recognition, vol. 10, pp. 318-323 (1998)

[24] Arandjelović, O., Cipolla, R.: An information-theoretic approach to face recognition from face motion manifolds. Image and Vision Computing 24, 639-647 (2006)

[25] Knappmeyer, B., Thornton, I.M., Bülthoff, H.H.: The use of facial motion and facial form during the processing of identity. Vision Research 43, 1921-1936 (2003)

[26] Baker, S., Matthews, I., Schneider, J.: Automatic construction of active appearance models as an image coding problem. IEEE Transations on Pattern Analysis and Machine Intelligence 26(10), 1380-1384 (2004)

[27] Cootes, T.F., Marsland, S., Twining, C.J., Smith, K., Taylor, C.J.: Groupwise diffeomorphic non-rigid registration for automatic model building. In: European Conference on Computer Vision, pp. 316-327 (2004) 
[28] Cootes, T.F., Twining, C.J., Petrovic, V., Schestowitz, R., Taylor, C.J.: Groupwise construction of appearance model using piece-wise affine deformations. In: British Machine Vision Conference, pp. 879-888 (2005)

[29] Fang, H., Costen, N.P.: Tracking face localization with a hierarchical progressive face model. In: Gonzàliz, J., Moeslund, T.B., Wang, L. (eds.) Tracking Humans for the Evaluation of their Motion in Image Sequences, pp. 89-99 (2008)

[30] Edwards, G.J., Lanitis, A., Taylor, C., Cootes, T.F.: Modelling the variability in face images. In: IEEE International Conference on Automatic Face and Gesture Recognition, pp. 328-333 (1996)

[31] Poh, N., Bengio, S.: Improving fusion with margin-derived confidence in biometric authentication tasks. In: Kanade, T., Jain, A., Ratha, N.K. (eds.) AVBPA 2005. LNCS, vol. 3546, pp. 474-483. Springer, Heidelberg (2005)

[32] Hanley, J.A., McNeil, B.J.: A method for comparing the areas under Receiver Operating Characteristic curves derived from the same cases. Radiology 148, 839$843(1983)$

[33] Hill, H., Johnston, A.: Categorizing sex and identity from the biological motion of faces. Current Biology 11, 880-885 (2001) 